\title{
Improving Efficiency By Using Synchronised Parallel Data Transmission Over Wsn
}

\author{
Rahul Bhandekar ${ }^{\text {a }}$, Monika Ingole ${ }^{\mathrm{b}}$, Anirudh Bhagwat ${ }^{\mathrm{c}}$, DR.Tryambak Hiwarkar ${ }^{\mathrm{d}}$ \\ ${ }^{a}$ Asst.Prof Dept of Computer Science \& Engg WCEM Nagpur. \\ ${ }^{\mathrm{b}}$ Asst.Prof Dept of Computer Science \& Engg WCEM Nagpur. \\ ${ }^{\mathrm{c}}$ Asst.Prof Dept of Computer Science \& Engg WCEM Nagpur. \\ ${ }^{\mathrm{d}}$ H.O.D Dept of Computer Science \& Engg WCEM Nagpur.
}

Article History: Received: 10 November 2020; Revised 12 January 2021 Accepted: 27 January 2021; Published online: 5 April 2021

\begin{abstract}
As the data transmission speed and the efficiency over the wireless network depend on the network or transfer device bandwidth. After physical implementation of wireless network which is difficult to dynamically control transfer in order to get high or low data transfer rate. Dedicating the fixed network for such a dynamic requirement network is not feasible. Many researchers are trying to enhance the wireless network speed by joining the transfer speed of multiple lines which will result in to asynchronous data transfer and data leakage. Hence the proposed system is to design and implements a dynamically controllable wireless network using the multiple radio frequency wireless devices. Here the proposed system will use multiple wireless devices and transfer data over multiple line depending upon the user configuration and synchronize the data over the receiving end. It will let the user control the wireless data transmission speed as per the requirement.
\end{abstract}

\section{Introduction}

Wireless Sensor Networks (WSNs) are a new class of networking technology that is increasingly becoming popular today. Huge strides taken in sensing technology, low power microcontrollers and communication radio have spurred the mass production of relatively inexpensive sensor nodes. Such large scale sensor networks far reimburse use of conventional networks in situations where terrain, climate and other environmental constraints obstruct the deployment and setting up of regular networks. Because of the tremendous scale at which such nodes can be deployed, they are extremely robust in terms of individual node failures which make them all the more favorable in such extreme situations. There has been an explosion in the use of sensor networks for environmental measurement and study. A range of applications have been built using sensor networks, from environmental monitoring to radiation detection to lots of tracking applications.

Broadly, sensor applications can be categorized into data gathering or tracking. Data gathering applications use sensor nodes to periodically measure the value of a particular environmental variable and recorded values are collected by a sink node for further processing. A WSN typically consists of a large number of low-cost, lowpower and multifunctional sensor nodes that are deployed in a region of interest. These sensor nodes are small in size but are equipped with sensors, embedded microprocessors and radio transceivers and thus they have not only sensing capability, but also data processing and communicating capabilities.

Sensor networks have the following unique characteristics and constraints:

(i) Dense node deployment

(ii) Battery powered sensor nodes

(iii) Severe energy, computation and storage constraints

(iv) Self configurable

(v) Unreliable sensor nodes

(vi) Frequent topology change

(vii) No global identification

(viii) Many to one traffic pattern 


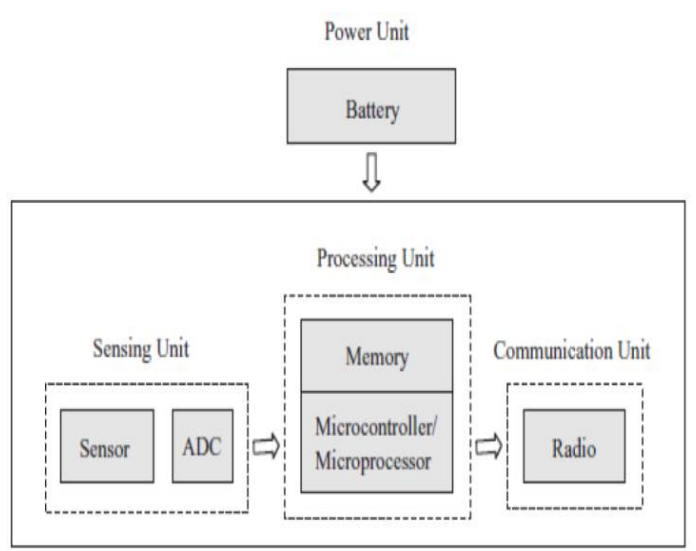

Fig (i): Sensor Node Structure

\section{Proposed Work}

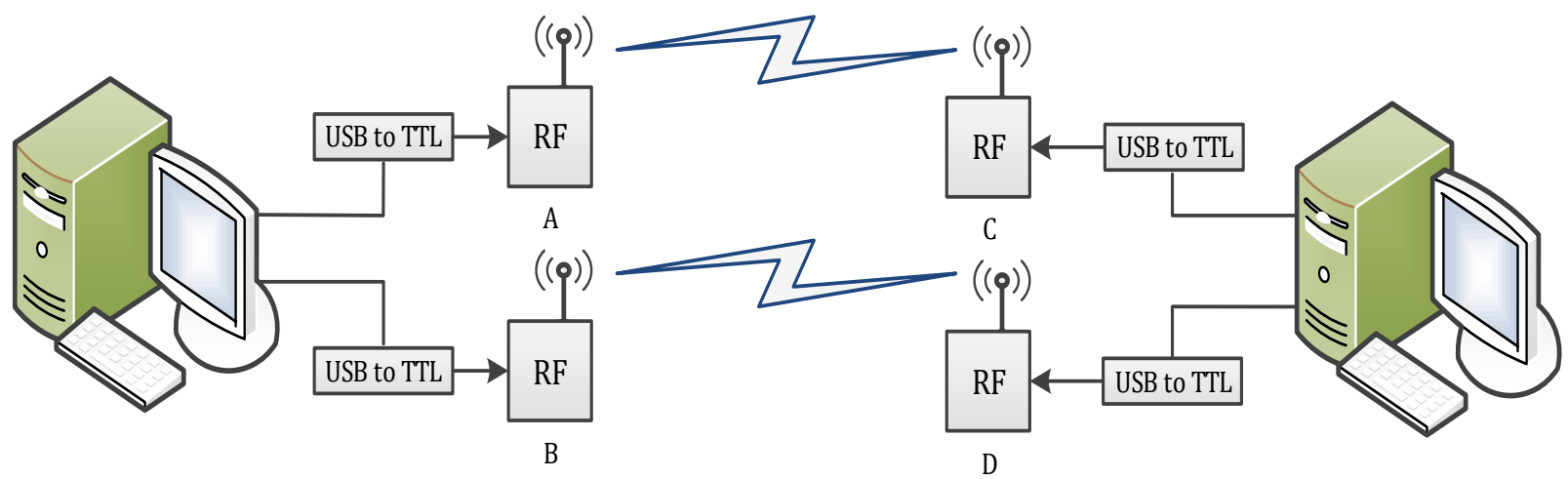

Building a wireless node with capability to broadcast a computer passed messages and receives the incoming messages.

1. Develop a windows based application to read and write data over the wireless devices.

2. Develop a windows based application to split the data or files in to multiple chunks or part and merge those parts back in to single file until the product is approved.

\section{Research Methodology}

\section{Transmission Modes}

Thetransmission mode refers to the number of elementary units of information (bits) that can be simultaneously translated by the communications channel. Infact, processors (and therefore computer sin general) never process (in the case of recent processors) as single bit at a time generally they are able to process several(most of the time It is 8:one byte), and for this reason the basic connections on a computer are parallel connections.

\section{Parallel Connection}

Parallel connection means simultaneous transmission of $\mathrm{N}$ bits. These bits are sent simultaneously over $\mathrm{N}$ different channels (a channel being,for example, a wire, a cable or any other physical medium).The parallel connection on PC-type computers generally requires 10 wires.

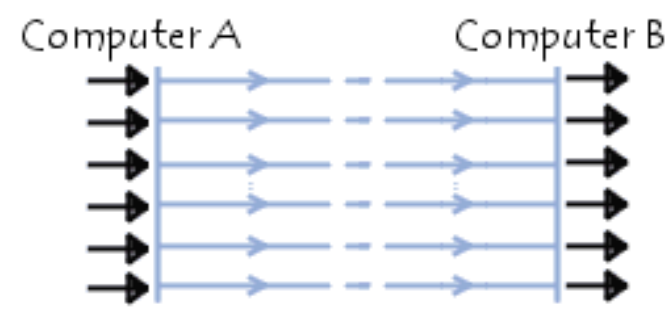

Parallel connection 


\section{Serial Connection}

In a serial connection, the data are sent one bit at a time over the transmission channel. However, since most processors process data in parallel, the transmitter needs

To transform in coming parallel data in to serial data and the receiver needs to do the opposite. These operations are performed by a communications controller (normally a UART(Universal Asynchronous Receiver Transmitter)chip).

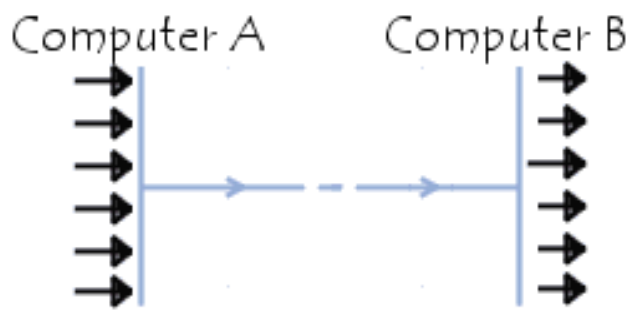

\section{Serial connection}

\section{Rf communication usingtransceiver:}

When time and RF engineering experience is of abundance, a designer may opt to use RF integrated circuits (chips or chipsets) to save on RF component costs. Using chips/chipsets, the designer actually develops the hardware and software workings of the product. While the individual chips/chipsets offer functionality the designer must dictate how those chips will work in concert with the software the designer will develop. This task is not for the faint of heart, as completed designs must also pass rigid regulatory testing for deployment in the various regions of the world. The regulatory approval process alone can become months or years of a cycle of rejection, followed by a reworking of the product and continued by rejection and reworking (transmitter / receiver) modules offer a faster time-to-market alternative to chips/chipsets that allow designers at all levels of RF experience to integrate a completed wireless system into their products. Many modules are manufactured as a drop-in solution where designers create a compatible pin-out on their processor board and supply serial data to the appropriate pins.

Modules offering the easiest integration allow the designer to send raw UART data into the module and expect that same data out on the receiving end of the wireless link.Wireless transceiver modules allowing the quickest time-to-market are also FCC and other regulatory agency approved. That means jurisdictions accepting FCC approval allow designers using FCC approved modules to bypass further testing for their wireless products. In Europe and other countries, pre-approved modules by ETSI and other regulatory bodies allow the designer to deploy products in various regions of the world with minimal additional approvals. The completed RF design and agency approval of many wireless transceiver modules make them a popular choice in the fast-paced world of wireless data communication product design.

\section{Simulation Result Using Ns2}

\section{Experimental Setup}

To evaluate the performance of the proposed system in WSNs, an extensive stimulation using Network stimulator 2.35 is performed. The simulation parameter is shown in Table below.

\begin{tabular}{|l|l|}
\hline PARAMETERS & VALUES \\
\hline Number Of Normal & 24 \\
\hline Simulation Area (M X & $1000 \times 1000$ \\
\hline Traffic Type & CBR \\
\hline Packet Size (Bits) & 512 \\
\hline Routing Protocol & DSR \\
\hline Queue Model & Priority Queue \\
\hline MAC & 802.11 \\
\hline Channel Type & Wireless Channel \\
\hline
\end{tabular}




\begin{tabular}{|l|c|}
\hline Antenna Type & Omni Antenna \\
\hline Performance Parameter & $\begin{array}{c}\text { Throughput,PDR, } \\
\text { Delay, Jitter }\end{array}$ \\
\hline
\end{tabular}

Table 3.5.1 Simulation Parameters

For the network set up, a set of 24 nodes are considered. The 24 nodes are as a normal nodes at the network. In our proposed system out of 24 nodes 12 nodes are act as a sender nodes and 12 nodes are receiver nodes. The connection is established through transceiver between sender and receiver nodes.

\section{Result Analysis}

\begin{tabular}{|c|c|c|c|}
\hline Nodes & $4-6$ & $4-4$ & $4-2$ \\
\hline $\begin{array}{l}\text { Thro uglpu } \\
\text { t (lhps) }\end{array}$ & 988.84 & $2^{553.4}$ & $\begin{array}{ll} & 494.2 \\
0 & \end{array}$ \\
\hline PDR & 77.26 & 76.12 & 74.55 \\
\hline Delay (ms) & 31.51 & 19.02 & 17.26 \\
\hline Jitter & 7.41 & 6.76 & 5.93 \\
\hline
\end{tabular}

Table 4.2.1 Result analysis

- Throughput

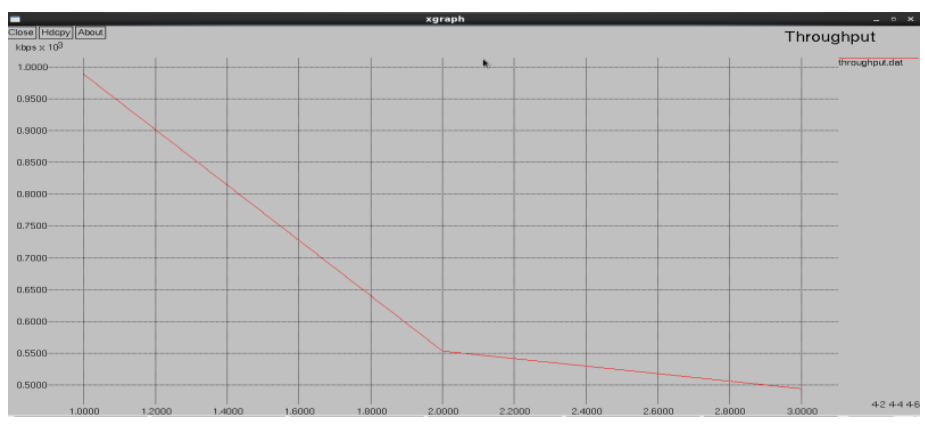

Fig 4.2.1 shows graphical analysis of the Table 4.4.1

The fig 4.2.1 shows the comparison of throughput of nodes 4-2, 4-4, 4-6. X-axis indicates Number of Nodes and $y$-axis indicates throughput values. As the no. of nodes decreases throughput values are also decreases .

- PDR 


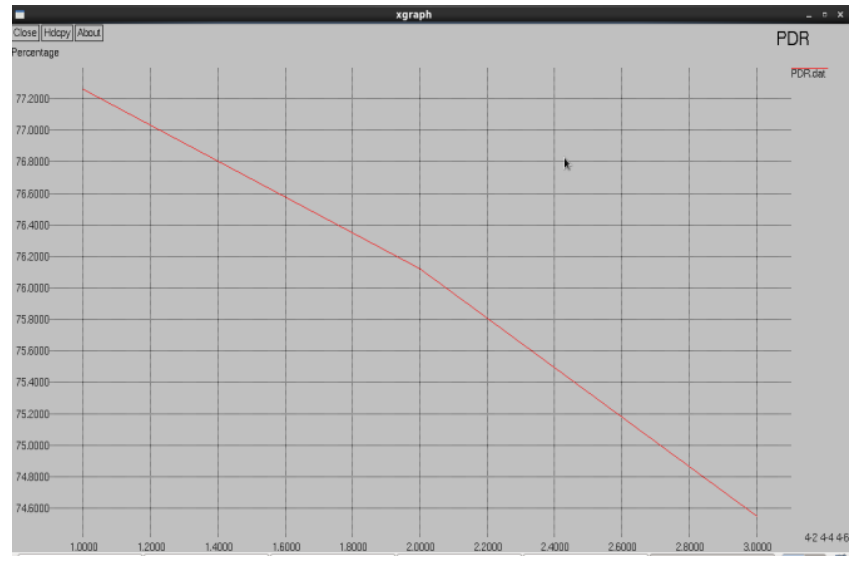

Fig 4.2.2 shows graphical analysis of the Table 4.4.1

The fig 4.2.2 shows the comparison of PDR of nodes 4-2, 4-4, 4-6. X-axis indicates Number of Nodes and yaxis indicates throughput values. As the no. of nodes decreases PDR Values are also decreases .

- Delay



Fig 4.2.3 shows graphical analysis of the Table 4.4.1

The fig 4.2.3 shows the comparison of Delay of nodes 4-2, 4-4, 4-6. X-axis indicates Number of Nodes and $y$-axis indicates Delay values. As the no. of nodes decreases Delay Values are also decreases.

- Jitter

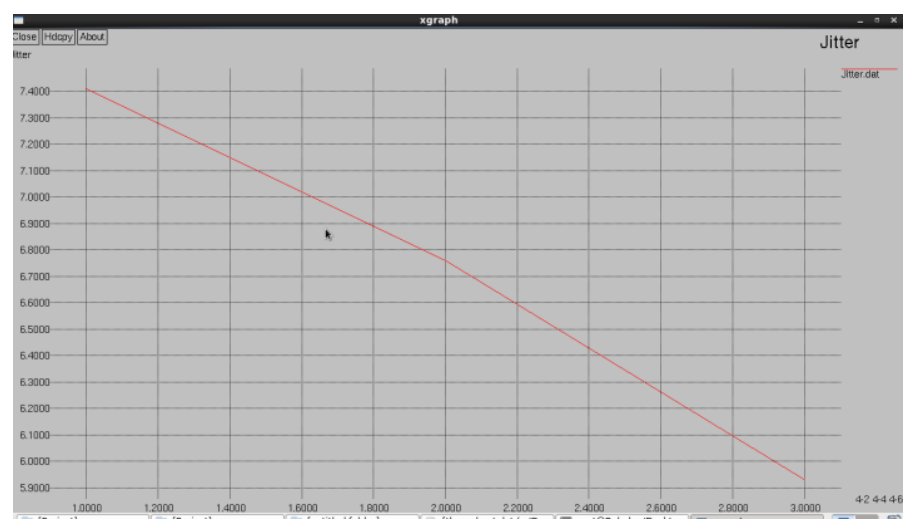

Fig 4.2.4 shows graphical analysis of the Table 4.4.1

The fig 4.2.4 shows the comparison of Jitter of nodes 4-2, 4-4, 4-6. X-axis indicates Number of Nodes and yaxis indicates Jitter values. As the no. of nodes decreases Jitter Values are also decreases

\section{Conclusion And Future Scope}

Large wireless sensor networks will be increasingly deployed in many application areas, among other improvements the individual node will offer increased storage and processing capacity. This implies that more data need to be transmitted in wireless sensor networks. More extensive computation tasks can be executed by 
sensor network, distributed storage and parallel processing will see rapid development. This research work focuses simultaneous transmission of data using multiple transceivers in wireless sensor networks. The system performance is assessed using three sets of transceivers as 4 sets of 2 transceivers, 4 sets of 4 transceivers, 4 sets of 6 transceivers. In the experiment, multiple wireless devices are used and data is transferred on multiple lines depending upon the user configuration and the data is synchronized at the receiving end. Through extensive simulation and result analysis we can conclude that the system performance improves for more simultaneous transmissions

\section{References}

Changqiao Xu, Member, IEEE, Tianjiao Liu, JianfengGuan,Hongke Zhang, and Gabriel-MiroMuntean, Member, IEEE, "CMT-QA: Quality-Aware Adaptive Concurrent Multipath Data Transfer in Heterogeneous Wireless Networks",IEEE Transactions on Mobile Computing, VOL. 12, NO. 11, NOVEMBER 2013.

Peng Li, Member, IEEE, and Song Guo, Senior Member, IEEE, "On the Multicast Capacity in EnergyConstrained Lossy Wireless Networks by Exploiting Intrabatch and Interbatch Network Coding” IEEE Transactions on Parallel and Distributed Systems, VOL. 24, NO. 11, NOVEMBER 2013.

Long Shi, Student Member, IEEE, Wei Zhang, Senior Member, IEEE, and Xiang-Gen Xia, Fellow, IEEE, “ Space-Time Block Code Designs for Two-User MIMO X Channels" IEEE Transactions On communications , VOL. 61, NO. 9, SEPTEMBER 2013.

Xuejun Tian, Yuguang Fang, Senior Member, IEEE, and Tetsuo Ideguchi, "MATS: Multichannel Time-Spread Scheduling in Mobile Ad Hoc Networks",IEEE TRANSACTIONS ON WIRELESS COMMUNICATIONS, VOL. 5, NO. 3, MARCH 2006.

T. Alpcan, J.P. Singh, and T. Basar, "Robust Rate Control for Heterogeneous Network access in Multihomed Environments," IEEE Trans. Mobile Computing, vol. 8, no. 1, pp. 41-51, Jan. 2009.

R. Fracchia, C. Casetti, C.F. Chiasserini, and M. Meo, "WiSE: BestPath Selection in Wireless Multihoming Environments,” IEEE Trans. Mobile Computing, vol. 6, no. 10, pp. 1130-1141, Oct. 2007.

T. Dreibholz, E.P. Rathgeb, I. Rungeler, R. Seggelmann, M. Tuxen,and R. Stewartm, "Stream Control Transmission Protocol: Past, Current and Future Standardization Activities," IEEE Comm. Magazine, vol. 49, no. 4, pp. 82-88, Apr. 2011.

T.D. Wallace and A. ShamiT, "A Review of Multihoming Issues Using the Stream Control Transmission Protocol,” IEEE Comm. Surveys and Tutorials, pp. 1-14, 2011.

R. Stewart, Q. Xie, M. Tuexen, S. Maruyama, and M. Kozuka, "Stream Control Transmission Protocol (SCTP) Dynamic Address Reconfiguration,” IETF RFC 5061, Sept. 2007.

J.R. Iyengar, P. Amer, and R. Stewart, "Concurrent Multipath Transfer Using SCTP Multihoming over Independent End-to-End Paths," IEEE/ACM Trans. Networking, vol. 14, no. 5, pp. 951-964, Oct. 2006.

P. Natarajan, N. Ekiz, P.D. Amer, and R. Stewart, "Concurrent Multipath Transfer during Path Failure," Computer Network, vol. 32, no. 15, pp. 1577-1587, Sept. 2009. 